Document downloaded from:

http://hdl.handle.net/10251/51177

This paper must be cited as:

Monzó Cárcel, PM.; Mogensen, P.; Acuña, J.; Ruiz Calvo, F.; Montagud Montalvá, Cl. (2015). A novel numerical approach for imposing a temperature boundary condition at the borehole wall in borehole fields. Geothermics. 56:35-44. doi:10.1016/j.geothermics.2015.03.003.

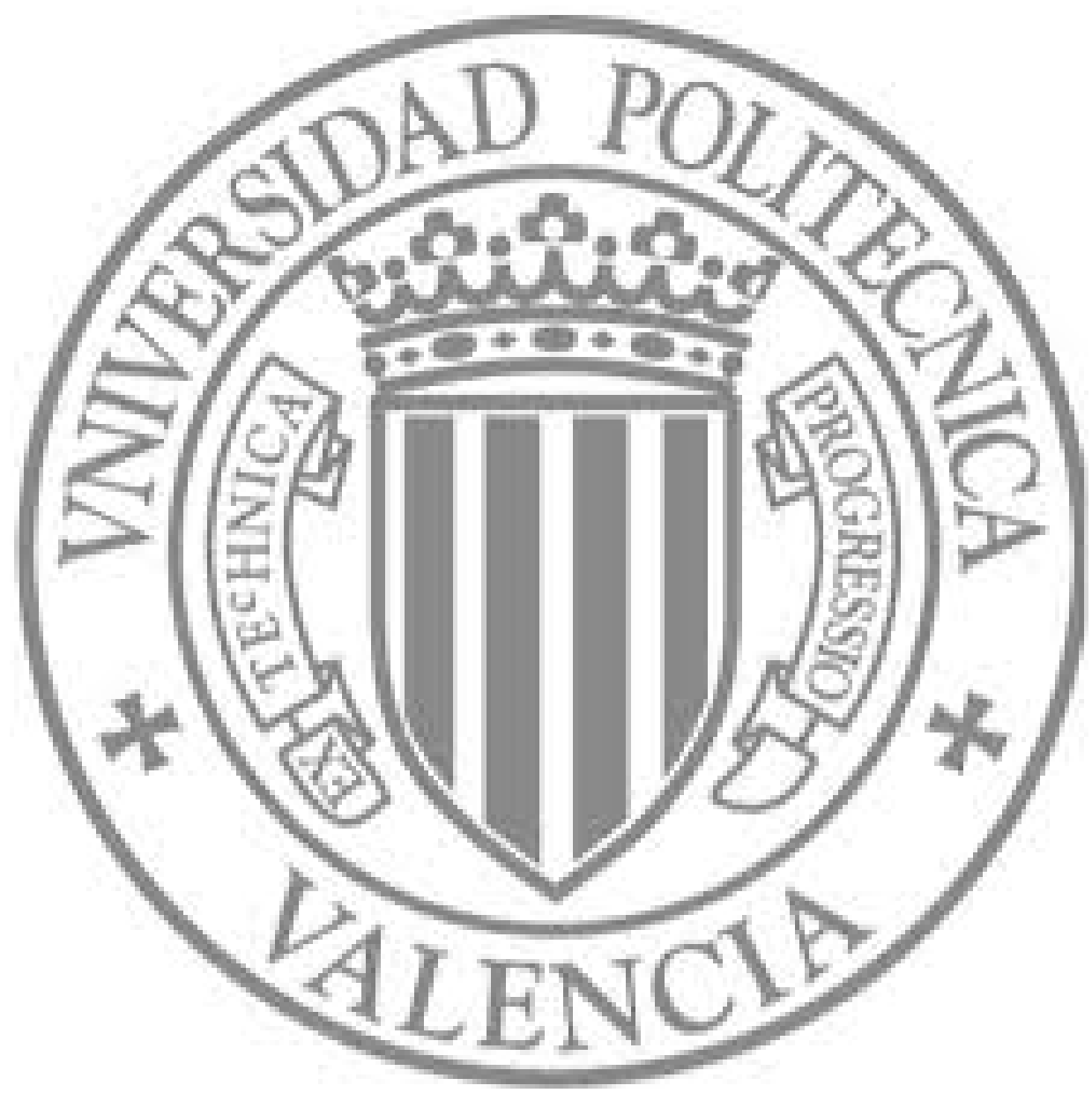

The final publication is available at

http://dx.doi.org/10.1016/j.geothermics.2015.03.003

Copyright Elsevier 


\title{
A NOVEL NUMERICAL APPROACH FOR IMPOSING A TEMPERATURE BOUNDARY CONDITION AT THE BOREHOLE WALL IN BOREHOLE FIELDS
}

\author{
Patricia Monzó *, Palne Mogensen, José Acuña, Félix Ruiz-Calvo, Carla Montagud \\ Energy Technology Department, KTH Royal Institute of Technology, Brinellvägen 68, 10044 \\ Stockholm, Sweden \\ *Corresponding author: Patricia Monzó \\ Telephone: +4687907450 e-mail address: patricia.monzo@energy.kth.se
}

\section{ABSTRACT}

The design of a borehole field should be based on a long-term simulation of its thermal response for the intended energy loads. A well-known method to evaluate the response is based on a pre-calculated dimensionless function, the g-function. When calculating g-functions, there are two commonly-used approaches for treating the boundary condition at the borehole wall: a constant heat flux at every instant of time, or a uniform temperature at a constant total heat flow to the borehole field. This paper is focused on a new approach to model the thermal process of borehole fields; in particular with a precise representation of a uniform temperature boundary condition at the borehole wall. The main purpose of this model is to be used as a research tool to either generate g-functions for particular cases or handle situations that cannot be addressed by others methods. First, the almost constant temperature along the borehole heat exchanger in operation requires a boundary condition of essentially isothermal boreholes along the depth. In a common case, the borehole heat exchangers are connected in parallel, thus all boreholes should have the same temperature. Also, the total heat flow to the borehole field should be constant over time. For this purpose, a numerical model in which the boreholes are filled with a hypothetical highly conductive material has been built, reproducing the isothermal condition. By thermally interconnecting the boreholes, the equal temperature condition is satisfied. Finally, the specified total heat flow is fed into one spot at the highly conductive material. The model is validated by generating g-functions of some simple borehole field configurations. The g-functions present, in general, a good agreement with the existing solutions for a similar boundary condition. Moreover, the model is also tested against real experimental data from a $2 \times 3$ borehole field at an office building. The simulated daily fluid temperatures are compared with measured daily fluid temperatures for the sixth year of operation. The simulated values present, in general, a good agreement with the measured data. The results show that there are no significant differences with regard to the boundary conditions at the borehole wall, which for this specific case is due to the fact that the system is thermally balanced.

\section{NOMENCLATURE}

$\alpha=$ Thermal diffusivity $\left(\mathrm{m}^{2} / \mathrm{s}\right)$

$\mathrm{B}=$ Borehole spacing $(\mathrm{m})$

$\mathrm{D}=$ Inactive upper part of the borehole $(\mathrm{m})$

$\mathrm{Fo}_{\mathrm{H}}=\alpha t / H^{2}(1)$ Fourier number, characteristic length $\mathrm{H}$

$\mathrm{H}=$ Active borehole length $(\mathrm{m})$ 
$\mathrm{k}=$ Thermal conductivity $(\mathrm{W} / \mathrm{m} \mathrm{K})$

$\mathrm{Q}=$ Total heat flow $(\mathrm{W})$

$q=$ Heat flow per unit length of borehole (W/m)

$\mathrm{r}_{\mathrm{b}}=$ Borehole radius $(\mathrm{m})$

$\mathrm{t}=$ Time $(\mathrm{s})$

$t_{S}=H^{2} / 9 \alpha(\mathrm{s})$ Characteristic time

$\mathrm{T}=$ Temperature $\left({ }^{\circ} \mathrm{C}\right)$

\section{ABBREVIATIONS}

$\mathrm{BC}=$ Boundary Condition

$\mathrm{BH}=$ Borehole

$\mathrm{BHE}=$ Borehole Heat Exchanger

BTES $=$ Borehole Thermal Energy Storage

$\mathrm{EED}=$ Earth Energy Designer Software

$\mathrm{FDM}=$ Finite Difference Method

$\mathrm{FEM}=$ Finite Element Method

FLS $=$ Finite Line Source

GCHP $=$ Ground-Coupled Heat Pump

GLHEPRO $=$ Professional Ground Loop Heat Exchanger Software

GSHP $=$ Ground-Source Heat Pump

$\mathrm{HCM}=$ Highly Conductive Material

SBM $=$ Superposition Borehole Model

$\mathrm{UPV}=$ Universitat Politècnica de València

\section{SUBSCRIPTS}

$\mathrm{b}=$ Borehole

$\mathrm{bw}=$ Borehole wall

$\mathrm{g}=$ Undisturbed

\section{INTRODUCTION}

During the last years, Ground-Source Heat Pump (GSHP) systems have become increasingly popular among the renewable technologies due to their high efficiency and their contribution to the mitigation of $\mathrm{CO}_{2}$ emissions. The most common GSHP systems in Sweden are those coupled to (nearly) vertical borehole heat exchangers (BHEs), the so-called Ground-Coupled Heat Pump (GCHP). In year 2012 there were a total of about 425,000 small (family houses) GCHP systems in Sweden, and 400 large Borehole Thermal Energy Storage (BTES) installations. While the growth rate of new small systems has slowed down, larger GSHP installations are nowadays starting to take a significant role in the Swedish low temperature heating energy supply (Andersson and Bjelm 2013).

GCHP systems are mainly based on an energy exchange from the building to the ground, or vice versa. A heat pump normally connects the indoor circuit of a building with the outdoor circuit on the ground side. The heat pump performance depends among other things on a proper design of the outdoor circuit, which commonly consists of a set of vertical heat exchangers buried in the ground. The design of 
large BHE fields should account for extrinsic factors such as a realistic assessment of the heating and cooling demand of the building, the temperature limitations on the fluids at the hot and cold side of the heat pump (HP), and the available drilling area. Moreover, intrinsic factors such as the thermal properties of the ground and the possible presence of groundwater flow should be evaluated. Taking all these factors into account, the design results in a borehole $(\mathrm{BH})$ field with a particular arrangement that comprises a certain number of BHs of prescribed lengths. A common procedure for analyzing the thermal response of a BH field is to use temperature response factors, so-called g-functions. The concept of the g-function was first presented in (Eskilson 1986) and (Eskilson 1987). The g-function is a thermal response factor for a specific geometry that relates the change of temperature at the $\mathrm{BH}$ wall, $T_{b}(\mathrm{t})$, over time with a constant average heat flow per unit BH length, $\mathrm{q}$, imposed from time $\mathrm{t}=0$. Equation 1 expresses the link between the g-function, the heat load, and the temperature, for a $\mathrm{BH}$ field installed in a ground with a thermal conductivity, k. $T_{g}$ is the undisturbed ground temperature. Only heat conduction is considered.

$$
T_{b w}(t)-T_{g}=\frac{q}{2 \pi k} * g\left(\frac{t}{t_{s}}, \frac{r_{b}}{H}, \frac{B}{H}, \frac{D}{H}\right)
$$

The g-function is a function of dimensionless variables, $\mathrm{r}_{\mathrm{b}} / \mathrm{H}$ and $\mathrm{B} / \mathrm{H}$, representing the aspect ratios to the active $\mathrm{BH}$ length $(\mathrm{H})$ of the $\mathrm{BH}$ radius $\left(\mathrm{r}_{\mathrm{b}}\right)$ and $\mathrm{BH}$ spacing $(\mathrm{B})$, respectively. The g-function depends also on a non-dimensional time $\mathrm{t} / t_{s}$ where $t_{s}$ is a characteristic time defined as the Fourier number with $\mathrm{H}$ as the characteristic length and $\alpha$ as the thermal diffusivity of the ground, that is $\mathrm{Fo}_{\mathrm{H}}=\alpha \mathrm{t} / \mathrm{H}^{2}$. The upper inactive part, $\mathrm{D}$, of the $\mathrm{BH}$, which could be the part above the groundwater level or an insulated casing was dismissed by Eskilson as a negligible parameter in the g-function definition. As indicated previously, the main use of the g-function is in the simulation of the long-term thermal response of a $\mathrm{BH}$ field exposed to variable heat loads. It should be said that a straight-forward calculation of this response for a multi-BH field with variable loading conditions is normally not within the capability of the BH field designer. To simulate such a thermal response, a temporal superposition of variable loads is applied to the g-function, as mathematically expressed in Equation 2. The variable load is split into $\mathrm{n}$ piecewise constant load steps $q_{i}$, starting at $t=0$ with $q_{1}$ lasting to $t_{1}$ where the next step $q_{2}$ starts and so on until the last step $\mathrm{q}_{\mathrm{n}}$ ending at $t_{\mathrm{n}}$. It should be noted that the geometrical aspect ratios have been omitted in the arguments of the g-function in Equation 2.

$$
T_{b w}(t)-T_{g}=\frac{1}{2 \pi k} *\left(q_{1} * g\left(\frac{t}{t_{s}},,\right)-q_{n} * g\left(\frac{\left(t-t_{n}\right)}{t_{s}},,\right)+\sum_{i=1}^{n-1}\left(q_{i+1}-q_{i}\right) * g\left(\frac{\left(t-t_{i}\right)}{t_{s}},,\right)\right)(2)
$$

This procedure is applied in well-known commercial software programs such as Earth Energy Designer (EED) (Hellström and Sanner 1994) and GLHEPRO (Marshall and Spitler 1994). When applying Equation. 1 and 2, in $\mathrm{BH}$ design, it is important to note that there is a temperature drop between the circulating fluid and the $\mathrm{BH}$ wall related to heat transfer resistances in and around the BHE. A library of g-functions for many relatively simple BH field geometries is implemented in these software programs. However, these two software codes are limited when it comes to specifying irregular BH field geometries. Moreover, the users can neither impose variable monthly heat loads over different years, nor daily or hourly loads. Due to restrictions at available drilling sites, many real installations require specific and unique designs of $\mathrm{BH}$ field configurations, which are not implemented in the library of the above mentioned software. This paper presents a novel numerical model in which special attention is given to impose a uniform temperature boundary condition (BC) at the $\mathrm{BH}$ wall. It can e.g. be used as a research tool to generate g-functions or to simulate directly the thermal response (fluid temperature) of a BH field. Examples are cases that other methods cannot address such as spatially varying ground properties or imposing variable thermal loads. The model was first tested by generating g-functions for some simple $\mathrm{BH}$ fields, a single $\mathrm{BH}$ and a $2 \times 3 \mathrm{BHs}$ configuration, and compared with some reference solutions. Moreover, the model was also tested to simulate daily fluid temperatures, which were compared with 
experimental data. The model was set up according to the geometrical characteristics and ground thermal properties at the Demo site at the Universitat Politècnica de València (UPV), Spain. For this purpose, the model first generated the g-function corresponding to the UPV BH field; then, the simulated daily temperatures were obtained by imposing the measured heat loads to the model.

\section{BACKGROUND}

The calculation of g-functions for multiple BH fields is a demanding and time consuming task. A variety of methods are in use, e.g. analytical, (semi)analytical and numerical, the latter based on Finite Difference (FDMs) and Finite Element Methods (FEMs). A crucial point when generating g-functions is the $\mathrm{BCs}$ at the $\mathrm{BH}$ wall, which requires two simultaneous conditions to be fulfilled. Firstly, all BHEs should receive a uniform inlet fluid temperature (They are in general hydraulically connected in parallel, so that they are also thermally connected). Secondly, the total heat flow injected to the BH field must be constant for the g-function generation. The reference solutions assume that the walls of all BHs have a uniform temperature, but changing over time as the ground responds to the heat load. The total heat load must be constant over time, meaning that the heat distribution between the BHs also changes with time as the BHs begin to thermally interact with each other.

Eskilson (1987) carried out an extensive work on generating g-functions by both numerical and analytical methods, in which the BCs at the $\mathrm{BH}$ wall also were studied deeply. The analytical method is based on the FLS and assumes constant heat flux at the BH wall. On the other hand, the numerical method is based on a FDM and allows imposing different BCs at the BH wall, from constant heat flux to uniform temperature. This numerical method is described in the Superposition Borehole Model (SBM) (Eskilson 1986). A constant heat flux BC is restricted to all BHs having the same heat flux at the $\mathrm{BH}$ wall, whereas a uniform temperature $\mathrm{BC}$ allows the heat flux at each $\mathrm{BH}$ to change in time while keeping the total heat flow constant. This may be the reason why the uniform temperature BC in SBM was preferred in the generation of the g-functions. The drawback of this method is a long computing time, especially for large BH fields. This is the reason for implementing a library of g-functions in commercial software. Although the library contains hundreds of g-functions for different multiple BH field configurations (most patterns are linear, square or rectangular), the users cannot always find a configuration that suits their needs. In case the pattern is found in the library, sometimes the aspect ratios of the BH field are different from those specified in the library for that prescribed pattern. Then, the software interpolates a g-function from a set of known g-functions contained in the library, which might lead to some loss of accuracy (Yang et al. 2010). The logarithmic interpolation often used may overestimate the g-function values by a few percent, $4 \%$ or less. (Malayappan and Spitler 2013).

As regards the analytical solution introduced by Eskilson, it was modified later by (Zeng et al. 2002). However, this solution was impractical, given the long computing time needed for the evaluation of a double integral expression, in both the space and time domain. (Lamarche and Beauchamp 2007) proposed a simplification in the time domain, converting the integration from a double to a simple expression, making it computationally faster. Recently, (Claesson and Javed 2011) have developed a simpler expression, encompassing both the short and long-term response. The long-term response is based on the analytical FLS solution. This solution also involves a simplification of the double integral, but in space domain. Thus, the temperature response is expressed as a single integral in time domain. The time derivative of the response temperature represents a weighting function for the preceding heat injections, which can be used to calculate the temperature for any $\mathrm{q}(\mathrm{t})$. This solution also accounts for the inactive part, D, of the BHEs.

The g-functions generated for identical $\mathrm{BH}$ configurations but using the uniform temperature (and constant total heat flow applied to the BH field) or the constant heat flux BCs show discrepancies that become more significant for large BH fields and large time scales. Among the FLS approaches, the one proposed by (Lamarche and Beauchamp 2007) overestimates the g-functions, whereas the solution proposed by (Claesson and Javed 2011) underestimates the g-functions, both in comparison with those 
generated from the numerical approach (Eskilson 1986). The advantage of the FLS approach is its flexibility to generate the g-function of any BHE configuration and the fast computation with an acceptable accuracy (Fossa 2011a and 2011b).

Particularly with analytical methods, the uniform temperature condition is difficult to manage and often constant heat flux at the $\mathrm{BH}$ wall is set as $\mathrm{BC}$ instead. Recent ways to handle the $\mathrm{BC}$ limitation of the analytical methods are presented in (Cimmino et al. 2013), (Lazarotto 2014). In those developments, the $\mathrm{BC}$ at the $\mathrm{BH}$ wall is set so that a constant heat flux is assumed along the $\mathrm{BH}$ wall, but the heat flow rate is not distributed equally among the $\mathrm{BHs}$ to fulfill an equal average temperature along the wall in all BHs. (Cimmino et al. 2013) uses response factors of borehole-to-boreholes and group-to-boreholes to account for the $\mathrm{BH}$ interactions and calculate the heat rate distribution among the BHs. The methodology has been implemented into MATLAB with a user interface that allows the calculation of the g-function for different BC, the so-called preprocessor (Cimmino and Bernier 2013). (Lazarotto 2014) connects BHs with each other, being able to account for the temporal variation of the heat load between BHs while keeping a constant total load and considering the FLS solution as of (Lamarche and Beauchamp 2007). In a close approximation to a more realistic BC in analytical approaches, (Cimmino and Bernier 2014) fulfills the uniform temperature condition at the $\mathrm{BH}$ wall by sectioning the $\mathrm{BHs}$ along the depth and solving the analytical equation (Claesson and Javed 2011) for each section. The uniform temperature condition is referred as BC-III in (Cimmino and Bernier 2014), its solution has also been implemented in the aforementioned preprocessor for the g-function generation.

In $\mathrm{BH}$ fields with both heat extraction and injection, repeated on a periodic, usually annual basis, only the net heat load will play a role in the long-term temperature response. In nearly balanced BH fields, the major part of the temperature response will thus be confined to the load variations within the yearly period and the corresponding part of the g-function, limited in a practical case to $\ln \left(9 \mathrm{Fo}_{\mathrm{H}}\right)<$ about -3 . This is where the differences between the discussed g-functions are small (Monzó et al. 2013b).

The numerical model presented in this paper imposes the isothermal condition at the $\mathrm{BH}$ wall, by filling the $\mathrm{BH}$ with a hypothetical highly conductive material (HCM). Moreover, the BHs are thermally connected to represent an equal inlet temperature condition while a constant total heat flow to the $\mathrm{BH}$ field is applied at one spot on the HCM. Moreover, the flexibility of the model allows imposing a vertical temperature gradient in the BHs, if so desired, by modifying the thermal conductivity of the HCM.

\section{NUMERICAL MODEL}

This section describes a FEM model in which the transient heat conduction problem in BH fields is studied from the BH wall out into the surrounding ground, a continuation of previous studies that were focused on the g-function generation by assuming constant heat flux at the BH wall (Monzó et al. 2013a and 2013b). The present model uses a more appropriate $\mathrm{BC}$ by imposing a uniform temperature at the $\mathrm{BH}$ wall and a uniform temperature along the $\mathrm{BH}$ length in all BHs.

\subsection{Thermal process around a single borehole}

First, the model is shown for the simplest case, a single BH in a 2D-axisymmetric space dimension with the $\mathrm{BH}$ aligned with the vertical axis. The active length, $\mathrm{H}$, of the $\mathrm{BH}$ is $100 \mathrm{~m}$ with a $\mathrm{BH}$ radius of $0.05 \mathrm{~m}$. A prescribed groundwater level at $4 \mathrm{~m}$ is considered as the inactive depth, D. The surrounding ground is represented by a square with sides of $1000 \mathrm{~m}$. It should be noted that the dimensions of the surrounding ground is not required to be as big as set out in our model. However, this model is being used to test other parameters and the authors prefer to keep this size in order not to compromise any results. Table 1 shows the aspect ratios for this single $\mathrm{BH}$ configuration. The thermal properties of the ground (granite) are defined in terms of thermal conductivity, $3.5 \mathrm{~W} / \mathrm{mK}$, specific heat at constant temperature, $850 \mathrm{~J} / \mathrm{kgK}$ and density, $2,600 \mathrm{~kg} / \mathrm{m}^{3}$. 
237

Table 1 : Geometrical aspect ratios of the single BH model

\begin{tabular}{cl}
\hline Geometrical Aspect Ratio & Value \\
\hline $\mathrm{r}_{\mathrm{b}} / \mathrm{H}$ & 0.00050 \\
$\mathrm{D} / \mathrm{H}$ & 0.040 \\
\hline
\end{tabular}

The initial condition is set to zero temperature in the whole domain and at all the boundaries. The $\mathrm{BCs}$ at all domain boundaries, except at the $\mathrm{BH}$ wall, are also fixed at zero temperature. This zero condition represents the undisturbed ground temperature.

The $\mathrm{BC}$ at the $\mathrm{BH}$ wall requires special attention. The heat transfer problem inside the $\mathrm{BH}$ is modeled by considering a hypothetical HCM, which is embedded inside the BH. The thermal conductivity of the $\mathrm{HCM}$ is chosen so that the temperature is almost uniform along the $\mathrm{BH}$ length to give a fair representation of the thermal gradient when the $\mathrm{BH}$ is in operation. After several iterations, the HCM thermal conductivity has been set to $10^{10} \mathrm{~W} / \mathrm{mK}$.

As regards the mesh, it consists of free triangular elements. Their size varies with smaller elements close to the BH and larger further out with around 30,000 elements in total. A transient study is set for a sufficiently long time in order to generate a g-function for values up to $\ln \left(9 \mathrm{Fo}_{\mathrm{H}}\right)$ around 2 , approximately equal to 170 years in this case. The computing time is about 20 minutes on a computer with 32 GB RAM and an Intel@ Xeon@ processor CPU E5-2620 at $2.00 \mathrm{GHz}$ (2 processors).

\subsection{Thermal process around a $2 \times 3 \mathrm{BHs}$ configuration}

The model becomes more complex when the number of BHs increases. The previous study of a single $\mathrm{BH}$ is here expanded to a multi-BH geometry, $2 \times 3 \mathrm{BHs}$ in a symmetric configuration. This model is built in a $3 \mathrm{D}$ space dimension. In this case, the $\mathrm{BH}$ dimensions are the same as in the single $\mathrm{BH}$ case with a $\mathrm{BH}$ spacing of $5 \mathrm{~m}$. Figure 1shows a representation of the modeled 3D domain. The total modeled domain is half the size of the BH field (heat transfer symmetry is applied to reduce the BH field by half), and the surrounding is $200 \times 200 \mathrm{~m}$ in the horizontal plane with $300 \mathrm{~m}$ depth. Table 2 shows the aspect ratios for this $\mathrm{BH}$ configuration.

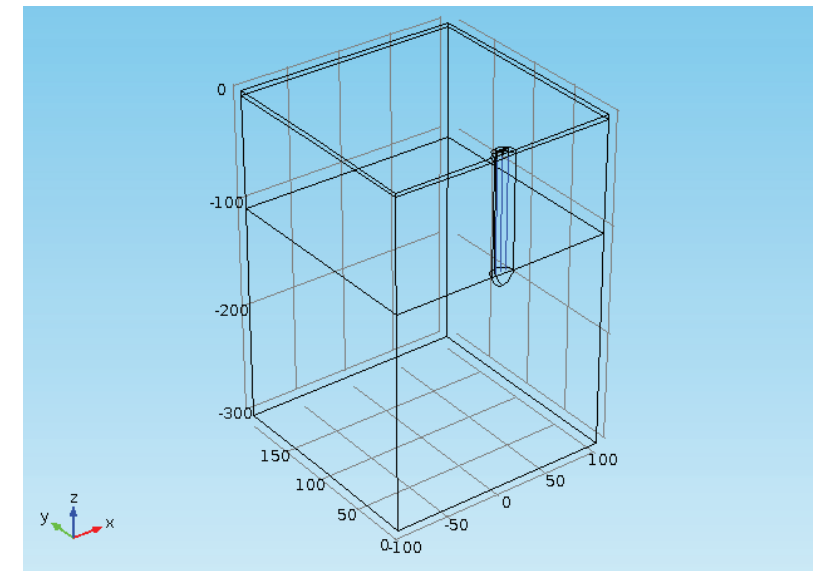

Figure 1 : Computational domain of $2 \times 3$ BHs configuration

Table 2 : Geometrical aspect ratios of the $2 \times 3$ BHs configuration

\begin{tabular}{cl}
\hline Geometrical Aspect Ratio & Value \\
\hline $\mathrm{r}_{\mathrm{b}} / \mathrm{H}$ & 0.00050 \\
$\mathrm{~B} / \mathrm{H}$ & 0.050 \\
\hline
\end{tabular}


The same considerations as for the single $\mathrm{BH}$ are taken with regard to the $\mathrm{BCs}$ and material properties. However, in this case, the uniform temperature $\mathrm{BC}$ at the $\mathrm{BH}$ wall in all the $\mathrm{BHs}$ has to be satisfied, i.e. all the BHs need to be thermally connected when the BH field comprises more than one BH. For that purpose, the BHs are physically connected with a bar of the same HCM material. This bar is connected to the HCM-filled BHs by cones of the same HCM. The cone element was chosen to connect the top of the active part of the borehole to the rod with a smooth diameter transition. The stepwise transition from the $\mathrm{BH}$ top to a slightly smaller cylindrical rod element first tested, gave rise to computational problems, since surface elements at the transition became smaller than the mesh size. Relevant surfaces of the rod and the cones are insulated. The heat load is applied on the top surface of the bar and is naturally distributed through the HCM material as governed by the thermal response of the ground, keeping a uniform temperature at all the BHs walls. Figure 2 shows a sketch of the connection between the BHs for the modeled geometry.

The HCM bar is elevated somewhat to stay off from the ground surface. The total length of the cone should be the sum of $\mathrm{D}$, the upper insulated part of the $\mathrm{BH}$, plus a certain elevation, $\mathrm{E}$, to ensure that the cone is embedded in the bar. Thus, the top side of the cone is embedded in the bar, whereas the bottom part of the cone is in contact with the top surface of the BH filling. The thermal conductivity of the HCM is again set to $10^{10} \mathrm{~W} / \mathrm{mK}$, and it applies to the bar, cones and inner domain of the BHs. No heat transfer occurs through the lateral surface of the cone. Table 3 shows the geometrical characteristics of the bar and cone.

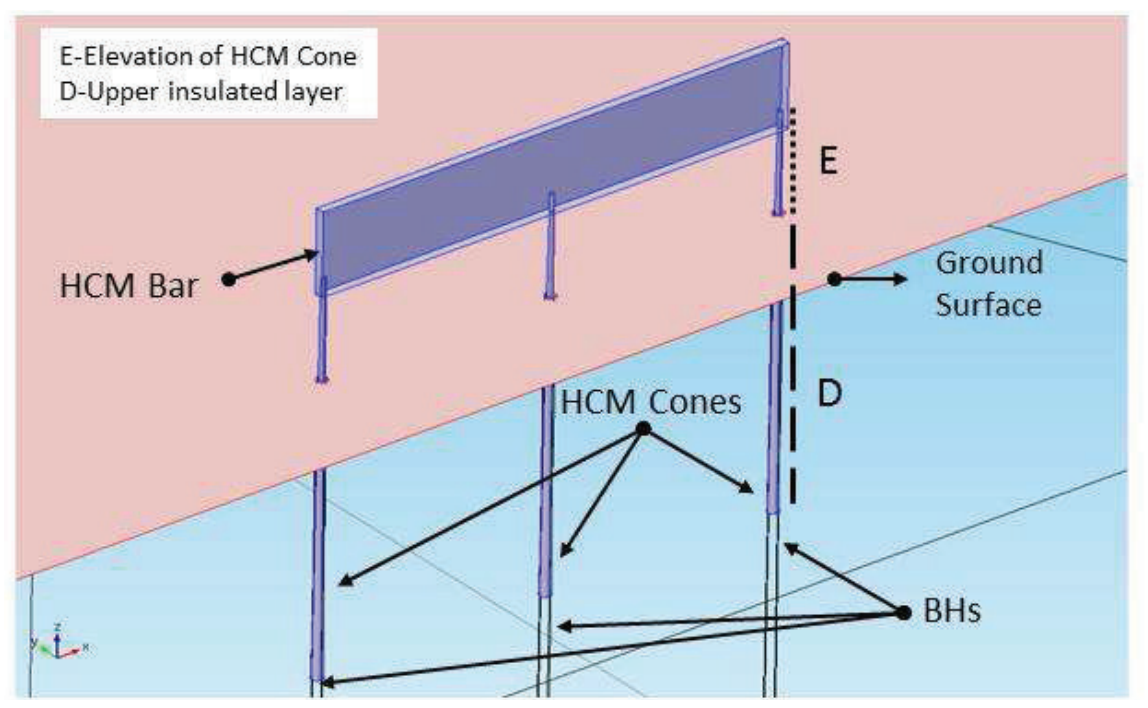

Figure 2 : Connection of the BHs to HCM bar. Half geometry of 2x3 BHs configuration

Table 3 : Geometrical characteristics of the bar and cones used to connect the BHs

\begin{tabular}{l|l|l}
\hline Element & Dimension & Size \\
\hline \multirow{3}{*}{ Bar } & Width [m] & 10.20 \\
& Depth [m] & 0.20 \\
& Height [m] & 1 \\
\hline \multirow{3}{*}{ Cone } & Bottom radius [m] & 0.05 \\
& Height [m] & 5.20 \\
& Top angle [deg] & $2 \times 0.5$ \\
\hline
\end{tabular}


Regarding the mesh, a free triangular mesh is defined on the layer at the upper part, D. This mesh presents small elements in the region close to the BHs, increasing with the distance from the BH field. Then, the triangular elements are swept along the BH length, creating a mesh of radial elements. The upper inactive part and the downward region of the $\mathrm{BH}$ field are meshed with tetrahedral elements. The bar, the cones and the inner part of the BHs are meshed in a similar way. The mesh consists of 250,000 elements. The model is set as a transient study for long term simulations, i.e. reaching values of $\ln \left(9 \mathrm{Fo}_{\mathrm{H}}\right)$ equal to around 2 (about 170 years for the values described in this section). The computing time is around 150 minutes on a computer with $32 \mathrm{~GB}$ RAM and an IntelC XeonC processor CPU E5-2620 at $2.00 \mathrm{GHz}$ (2 processors).

\section{4. g-function GENERATION}

In Figure 3and Figure 5, the g-functions generated from our numerical model are compared with gfunctions obtained using analytical FLS solutions (constant heat flux at the BH wall), labelled as "FLSConstant q", as well as those generated using commercial tools based on SBM with uniform temperature BC. i.e. EED or GLHEPRO, referred as "FDM-Uniform T" in this paper. A third analytical solution that adapts the FLS approach to the uniform temperature condition, referred as BC-III in (Cimmino and Bernier, 2014) is also included in the comparison, labelled as "FLS-Uniform T" in this paper. The results calculated from our numerical model are identified as "FEM-Uniform T".

\section{1 g-function for a single borehole}

A constant heat flow is applied during the whole period of interest to generate the g-function. Figure 3 shows the g-functions for the single $\mathrm{BH}$.

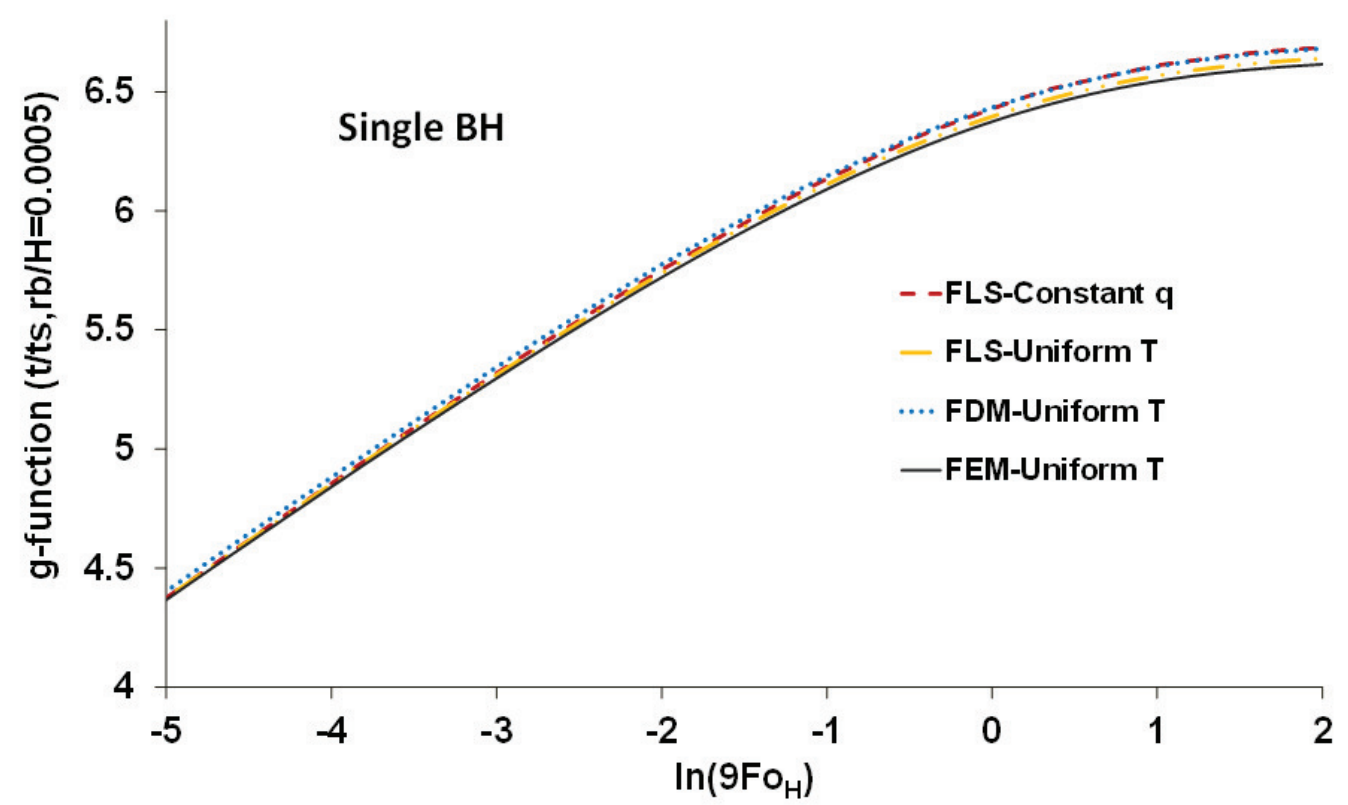

Figure 3 : g-functions for a single $\mathrm{BH}$

The "FEM-Uniform T" presents, in general, a good agreement with those solutions generated with a similar BC at the BH wall, "FDM- Uniform T" and "FLS- Uniform T". In the asymptotic part of the curve, at about $\ln \left(9 \mathrm{Fo}_{\mathrm{H}}\right)=2$ or 170 years, for the given parameters in this paper, the differences between 
"FDM-Uniform T" and "FEM-Uniform T" solution are around 0.07 units. In the beginning $\ln \left(9 \mathrm{FO}_{\mathrm{H}}\right)=-4.5$ or 90 days approx., the difference becomes around 0.04 units. For the given value of the thermal conductivity and a constant heat injection of $20 \mathrm{~W} / \mathrm{m}$, the temperature differences are about 0.035 and $0.06 \mathrm{~K}$ for 90 days and 170 years, respectively. The "FEM-Uniform T" solution increases around $0.3 \%$ when the ratio $\mathrm{D} / \mathrm{H}$ varies from 0.04 to 0.05 for a value of $\ln \left(9 \mathrm{Fo}_{\mathrm{H}}\right)$ equal to 2 and $\mathrm{H}=100 \mathrm{~m}$. The "FLSConstant q" solution comes out between the "FDM-Uniform T" and "FEM-Uniform T" g-functions. In comparison with "FDM-Uniform T", "FLS-Constant q" deviates around 0.02 units in the beginning. For the single $\mathrm{BH}$, no major differences are found when changing the $\mathrm{BC}$. All solutions are in general the same and all of them consider the finite length of the $\mathrm{BH}$, though the effects at both $\mathrm{BH}$ ends are different. The "FLS-Constant q" solution, however, is different at shorter times, given that it is based on a line source at the BH center and not a source at the $\mathrm{BH}$ wall. All curves meet at about $\mathrm{t}=5 \mathrm{r}^{2} / \alpha\left(\ln \left(9 \mathrm{Fo}_{\mathrm{H}}\right)=-\right.$ $11.39)$ and even with a smaller error at $\mathrm{t}=20 \mathrm{r}^{2} / \alpha,\left(\ln \left(9 \mathrm{Fo}_{\mathrm{H}}\right)=-10.01\right)$. This time period is not included in Figure 3. This situation will obviously become different for multiple BHs when BH interaction affects the validity of the constant heat flux $\mathrm{BC}$ more strongly than for a single $\mathrm{BH}$.

The validity of the imposed $\mathrm{BC}$ (uniform temperature) and the behavior of the heat flux have been observed in detail. Figure 4 shows the temperature and linear heat flow profiles for different instant of time at a cut line located $0.005 \mathrm{~m}$ outside the $\mathrm{BH}$ wall.

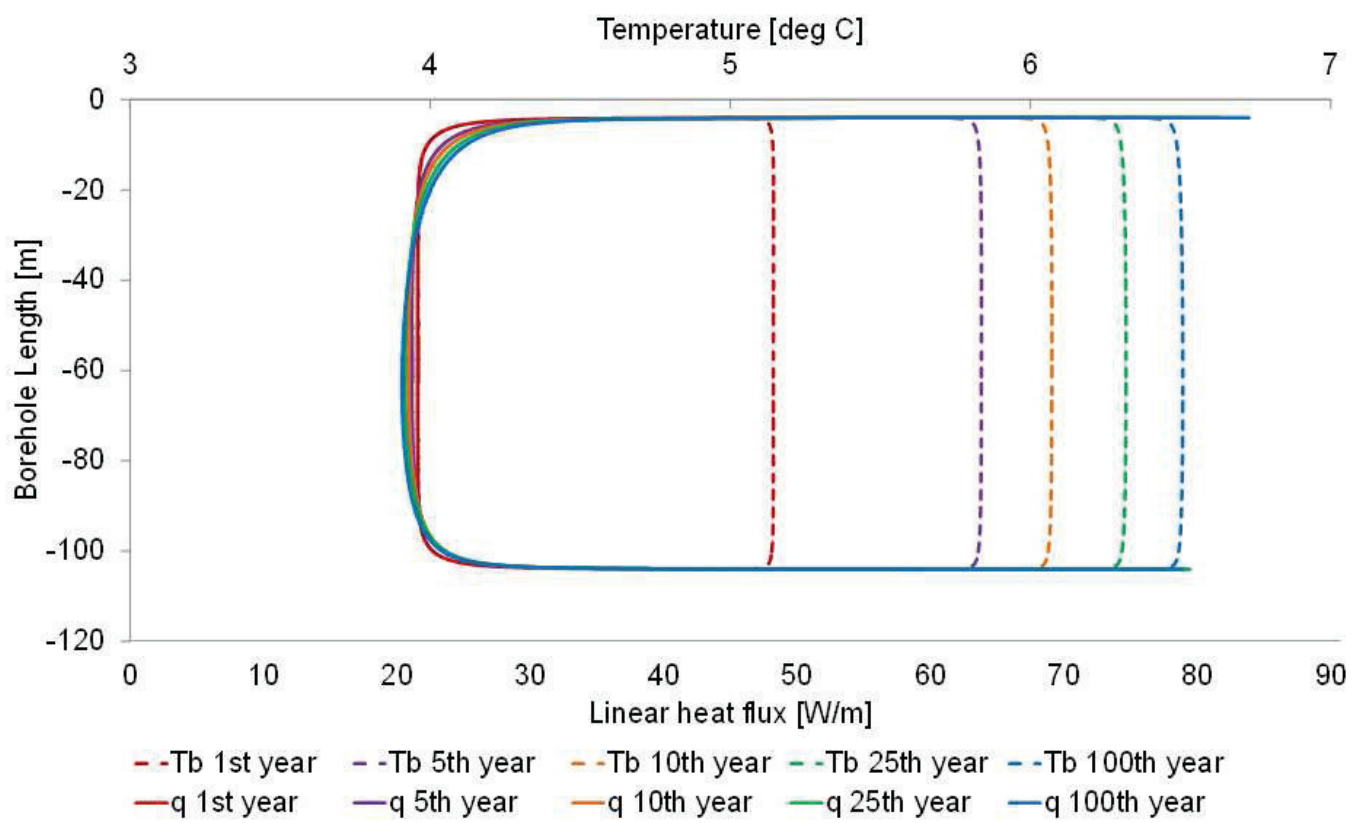

Figure 4 : Temperature and linear heat flux along the $\mathrm{BH}$ wall in a single $\mathrm{BH}$ 
During the whole simulation period, the temperature profile is uniform along the $\mathrm{BH}$ length. Since the temperature evaluations, due to technical reasons, are carried out at a parallel line $0.005 \mathrm{~m}$ outside the $\mathrm{BH}$ wall, some little deviations are appreciated at the top and at the bottom. The axis referred to the linear heat flux varies from 0 to $90[\mathrm{~W} / \mathrm{m}]$ in order to show how the heat dissipates at the top and the bottom of the BH. As shown in Figure 4, more heat is dissipated at the top part of the BH field than at the bottom part which results from the thermal process and BCs defined on the ground domain. A zero temperature $\mathrm{BC}$ at $\mathrm{z}=0$ may be modeled by a mirror image of the $\mathrm{BH}$ field, with opposite temperature and with the ground as mirror. The top of the BHs are of course closer to the mirror tops than the bottom ends are to the mirror image of them. Thus the temperature field is stronger at the surface than at the bottom, which explained the behavior observed in Figure 4.

\section{2 g-function generation for a $2 \times 3$ BHs configuration}

Figure 5 shows the g-function for the $2 \times 3$ BHs configuration. As in the case of the single $\mathrm{BH}$, Figure 5 includes the $\mathrm{g}$-functions from the reference solutions which are labelled as described at the beginning of this section, "FLS-Constant q", "FLS-Uniform T" and "FDM-Uniform T". The "FLS-Constant q", "FLSUniform T" solutions and our "FEM-Uniform T" solution are calculated with the aspect ratio $\mathrm{D} / \mathrm{H}=0.04$. However, the "FDM-Uniform T" solution, as mentioned above, does not clearly specify the value assumed for $\mathrm{D} / \mathrm{H}$.

The g-functions calculated from the four approaches present small deviations in the beginning when $\ln \left(9 \mathrm{FO}_{\mathrm{H}}\right)<-2$, that is about 3 years (for the ground thermal properties described in section 3.1). In general, the "FEM-Uniform T" shows a good agreement with "FDM-Uniform T" and the "FLS-Uniform T", presenting lower g-values. SBM solutions ("FDM-Uniform T" solution in this paper) are usually used as a benchmark. Even though SBM solutions have not been exhaustively validated, experimental validation (Cullin et al., 2013, Cullin, 2014 and Cullin et al., 2015) have demonstrated that they have acceptable accuracy. The slight deviation of "FEM-Uniform T" from "FDM-Uniform T" is about 0.16 units in the beginning $\left(\ln \left(9 \mathrm{Fo}_{\mathrm{H}}\right)=-4.5\right)$. The difference increases up to 0.28 units in the asymptotic part $\left(\ln \left(9 \mathrm{Fo} \mathrm{H}_{\mathrm{H}}\right)=2\right)$. In terms of temperature differences, for a thermal conductivity of $3.5 \mathrm{~W} / \mathrm{mK}$ and a constant heat injection of $20 \mathrm{~W} / \mathrm{m}$, the "FEM- Uniform T" deviates from "FDM-Uniform T" about 0.14 and $0.26 \mathrm{~K}$ for $\ln \left(9 \mathrm{Fo}_{\mathrm{H}}\right)=-4.5$ and 2 respectively. In the asymptotic part of the curve $\left(\ln \left(9 \mathrm{Fo}_{\mathrm{H}}\right)=1.5\right)$, the "FEM-Uniform T" g-function increases around $1.01 \%$ when the ratio $\mathrm{D} / \mathrm{H}$ varies from 0.04 to 0.05 for $\mathrm{H}=100 \mathrm{~m}$.

The "FLS-Constant q" is very close to the "FDM-Uniform T" in the beginning, slowly reaching a deviation from "FDM-Uniform T" of 0.06 units at $\ln \left(9 \mathrm{Fo}_{\mathrm{H}}\right)=-2$. Thereafter the deviation increases at a faster rate reaching 0.60 units near the asymptotic end of the curve whereas the "FLS-Constant q" solution comes out significantly higher, as described in section 2 . 


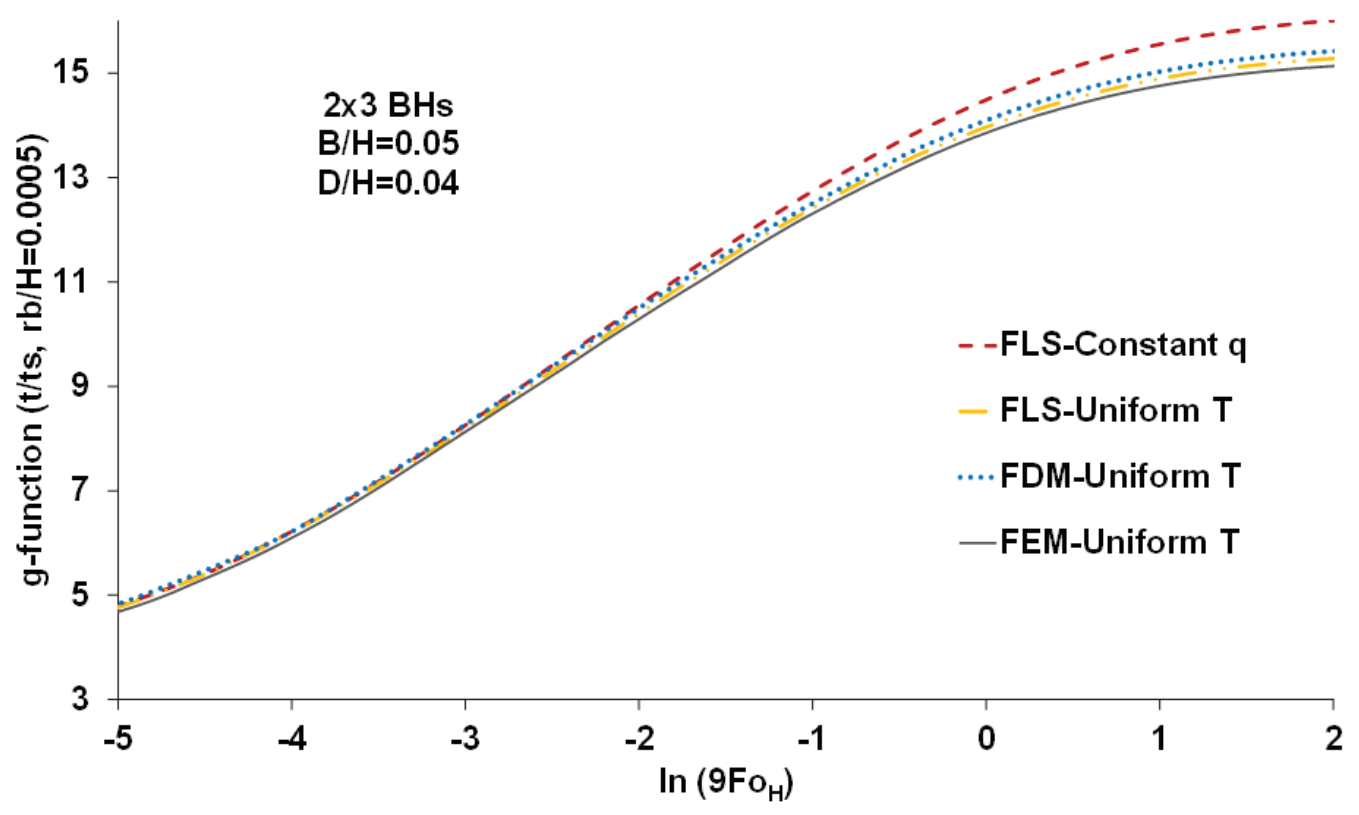

Figure 5 : g-function for a $2 \times 3 \mathrm{BHs}$ configuration

In general, in comparison with "FDM-Uniform T", the g-functions from "FEM-Uniform T", both single borehole and $2 \times 3$ BHs configurations; present a good agreement with slightly lower g-values. The g-function obtained from "FEM-Uniform T" presents small deviations both for the single BH and for the $2 \times 3 \mathrm{BHs}$ configuration. These differences are partly related to the different value of $\mathrm{D} / \mathrm{H}$ considered in "FDM-Uniform T" and "FEM-Uniform T". As mentioned above the aspect ratio D/H is not clearly specified in the solutions "FDM-Uniform T. The differences are also partly attributed to some adjustable features in the mesh and post-processing tools of our numerical model. Using "FDM-Uniform T" as an absolute reference could also be questioned.

To check that the uniform temperature condition is also satisfied in our model for the $2 \times 3 \mathrm{BHs}$ configuration, the average heat flow and average temperature profiles of the inner and the outer BHs are calculated. This is illustrated in Figure 6. These profiles have been calculated by creating a cylindrical parameterized surface to the BH wall, of both the inner and outer BHs. This surface is at a distance of $\mathrm{r}_{\mathrm{b}}+$ $0.0025 \mathrm{~m}$ from the BH center. Since the thermal response of the symmetric BHs is the same, Figure 6 only shows the thermal process of one inner $\mathrm{BH}$ and one outer $\mathrm{BH}$.

As shown in Figure 6, the average temperatures in both inner and outer BHs are the same at all instants of time and heat transfer at the inner $\mathrm{BH}$ is significantly lower in comparison to the outer $\mathrm{BH}$. In the first month, the differences in the heat transfer between inner and outer BHs are very small, barely 40 $\mathrm{W}(0.6 \%)$. Then, the differences between the average heat flow at the inner and outer BHs start to become more pronounced, increasing progressively up to approximately $484 \mathrm{~W}(7.3 \%)$. Thenceforward, this difference is almost constant, except for some fluctuations, which are attributed to the solver, as explained below. The average temperature is kept equal in the inner and outer BHs as expected. The rearrangement of the power partition between inner and outer BHs occurs in about two years. Figure 6 confirms that in our approach the heat flow is not distributed equally among the BHs in order to satisfy a uniform temperature condition. The quotient of the heat flow from the outer BHs to the average is around 1.07, whereas the quotient of the inner BHs is about 0.85. Similar differences in heat distribution are discussed in (Cimmino and Bernier, 2014). 


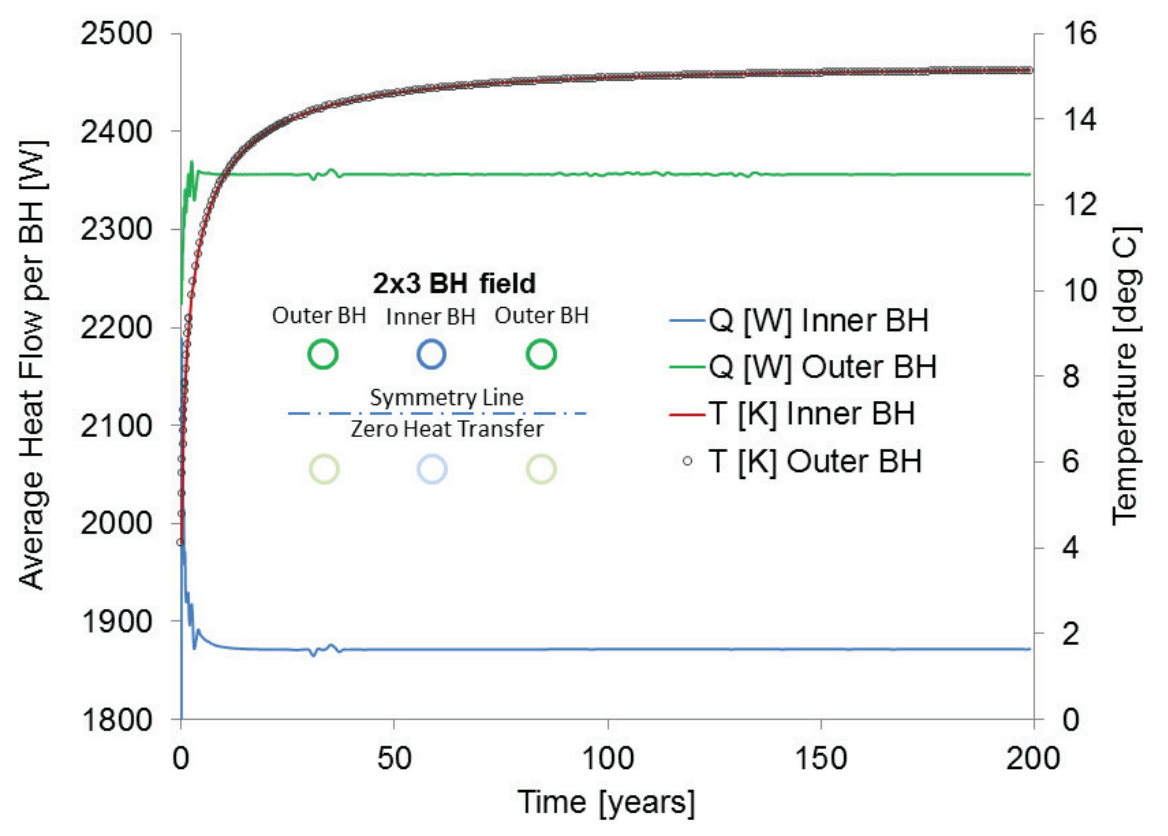

Figure 6 : Average Heat Flow and Average Temperature in inner and outer BHs (2x3 BH configuration).

In Figure 6, during the first 4 years, the average heat flow, of both inner and outer BHs, present some fluctuations. These fluctuations are attributed to the very short time step taking by the solver to calculate the solution in this period, which starts on an initial time of $0.001 \mathrm{~s}$ and increases up to a maximum of two months and averages the solutions for the output interval of time in this period (1 month), as set in the features of the solver. Other fluctuations can also be seen in the interval between 26 and 37 years, which are due to the time step set in the solver, as explained below. The average heat flow of the outer $\mathrm{BH}$ also shows some small fluctuations when the time interval is approximately between 90 and 140 years. All these fluctuations can be attributed to the maximum time step (2 months) selected in the solver and the time intervals in which the solution is given as output. A short output interval is chosen for the first 25 years, increasing from one to several months as the simulation proceeds. After the first 25 years, the output is written every year. The condition of the maximum time step is satisfied during the whole simulation period. Despite these fluctuations, the reader can easily appreciate the thermal behavior of both the inner and outer BH.

Moreover, the numerical model allows us to study in detail the heat transfer over the whole surface of the BH wall and any other point in space. The heat flux and temperature are studied for both inner and outer BHs along a line, $0.005 \mathrm{~m}$ outside the $\mathrm{BH}$ wall in Figure 7 and Figure 8.

As shown in Figure 7 and Figure 8, the temperature profiles at the inner and outer BHs are uniform. Both inner and outer BHs present the same temperature profiles for all instants of time, which confirm the uniform temperature condition that is imposed in our approach. The heat flux profiles are different for the inner and the outer BHs, as expected. However the heat distribution between the BHs changes with time. The linear heat flux profiles changes as the BHs thermally interfere with each other and with the surroundings. Thus, the linear heat flux at the inner and the outer BH is very similar during the first year, around 18 and $21 \mathrm{~W} / \mathrm{m}$, respectively at the central part of the BH. But as time goes, the differences become more significant, less heat is dissipated by the inner BH, i.e. around $15 \mathrm{~W} / \mathrm{m}$ at the central part of the $\mathrm{BH}$ for the $25^{\text {th }}$ year of operation, whereas the linear heat flux is around $18 \mathrm{~W} / \mathrm{m}$ at the central part of the outer $\mathrm{BH}$ for the same operation time. Due to the short distance between the $\mathrm{BH}$ top and the oppositely charged mirror $\mathrm{BH}$ above ground in comparison with the bottom part and its mirror image, 
more heat is dissipated at the top part of the BH in both inner and outer BHs. High values are observed at the bottom and at the top of both inner and outer BHs, as in the case of a single $\mathrm{BH}$.

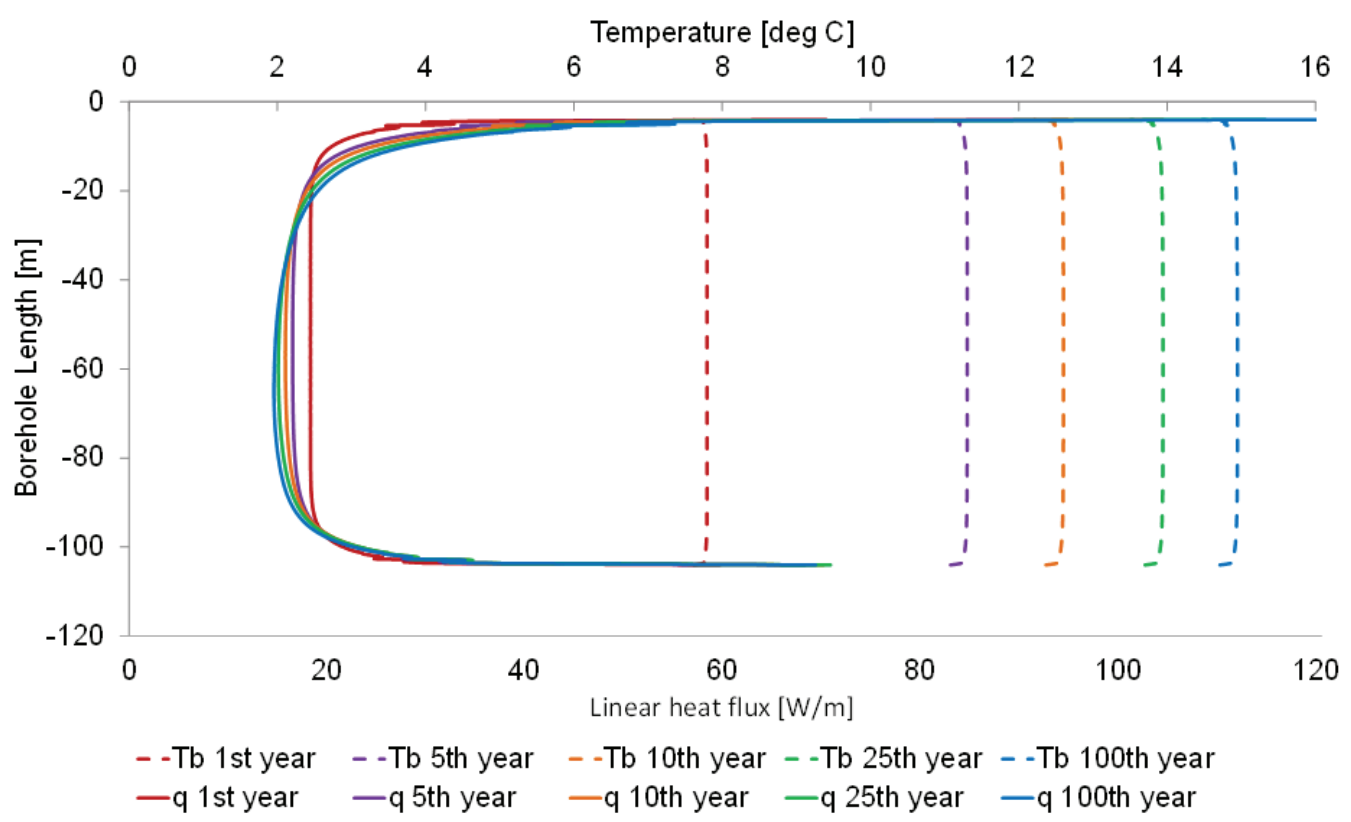

Figure 7 : Temperature and linear heat flux at the inner $\mathrm{BH}(2 \times 3 \mathrm{BH}$ configuration)

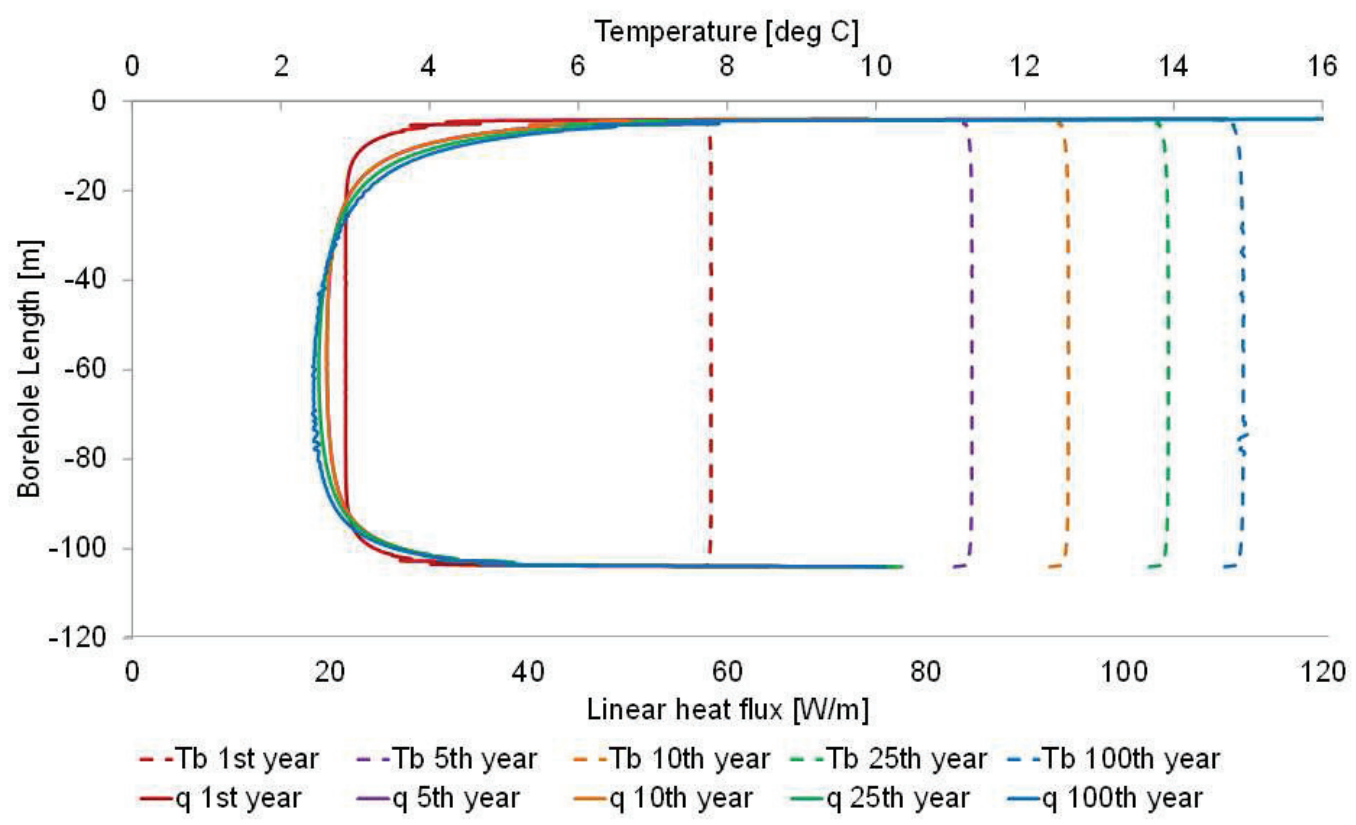

Figure 8 : Temperature and linear heat flux at the outer $\mathrm{BH}(2 \times 3 \mathrm{BH}$ configuration)

Figure 9 shows the linear heat flux profile at the inner and outer BHs for the 1st and the 25th years of operation. It can be observed the differences on the linear heat flux profiles between the inner and the outer BH. The outer BH presents higher linear heat flux for both the 1st and the 25th years than the inner $\mathrm{BH}$, being more significant in the last period. 


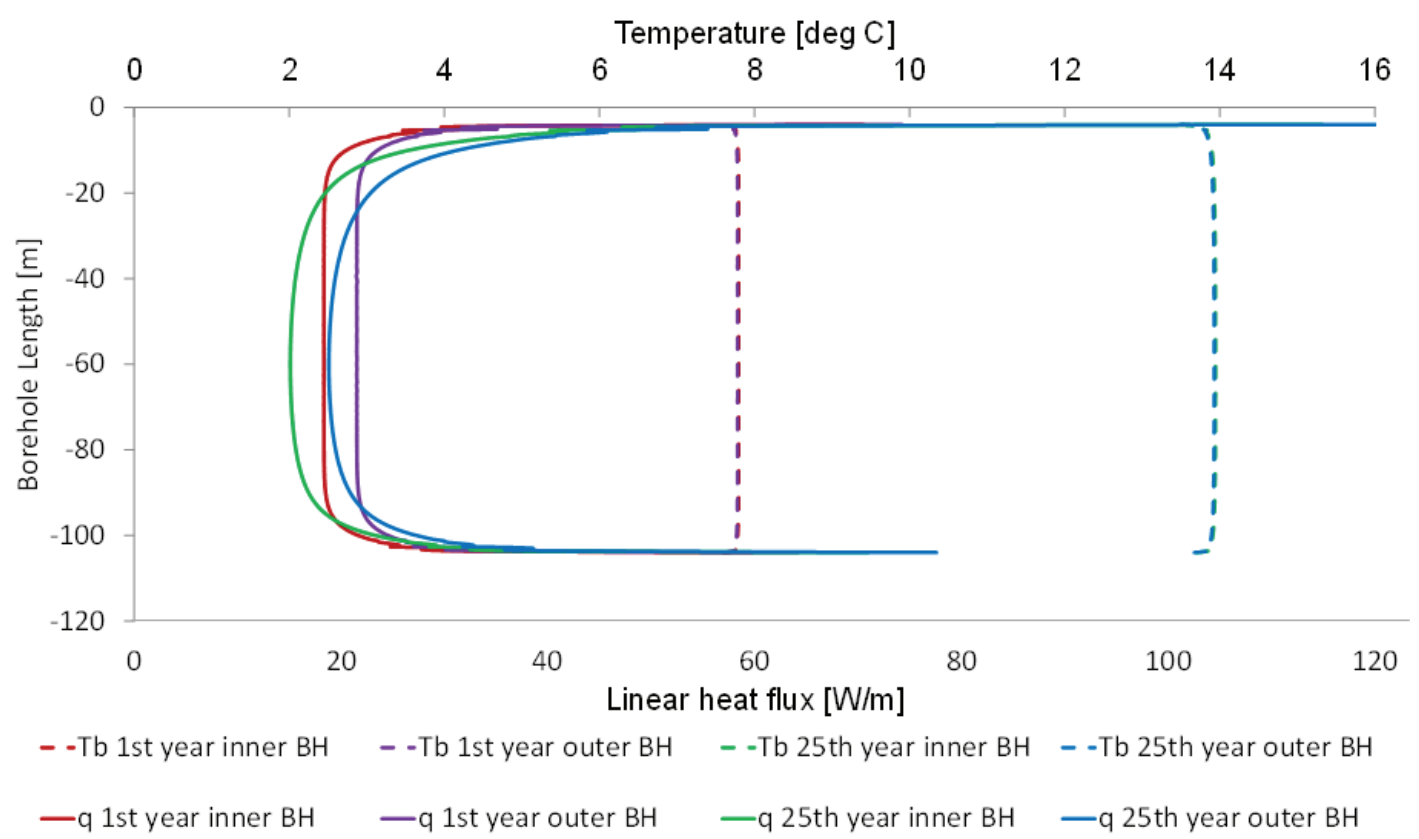

Figure 9: Temperature and linear heat flux at the inner and the outer $\mathrm{BHs}(2 \times 3 \mathrm{BH}$ configuration)

\section{EXPERIMENTAL VALIDATION}

\subsection{Description of the ground heat pump installation}

The GSHP installation analyzed below was built in the framework of a European FP5 project called, GeoCool at the UPV, Valencia, Spain. The system has been in operation since February 2005 for the air conditioning of a set of offices at the Department of Applied Thermodynamics. The installation has been fully monitored since then. The system is programmed to operate between 07:00 and 22:00 $\mathbf{h}$ in summer and between 06:00 and 21:00 h in winter, 5 days per week. The heating season starts in November and ends in April, while the cooling season comprises the period from May to October. A more detailed description of this GSHP system has been presented in previous publications by Montagud et al. (2011), Corberán et al. (2011).

The system consists of a reversible water-to-water heat pump, with a nominal cooling capacity of 16 $\mathrm{kW}$ and a nominal heating capacity of $19 \mathrm{~kW}$. The heat pump allows the heat exchange between two main circuits: the internal circuit, consisting of 12 parallel connected fan coils, a water storage tank and an internal hydraulic loop and the external circuit consisting of a set of BHEs. The thermal loads and water temperature data used in this paper are collected from sensors in the external circuit.

The $\mathrm{BH}$ field consists of 6 vertical $\mathrm{BHs}$, arranged in a $2 \times 3$ rectangular grid, with a separation between BHs of $3 \mathrm{~m}$. Each BH is $51 \mathrm{~m}$ deep with $1 \mathrm{~m}$ of header depth, and has a diameter of $150 \mathrm{~mm}$. The groundwater level is about $3.5 \mathrm{~m}$. The BHs contain a single HDPE U-tube $32 \times 3.3 \mathrm{~mm}$ with a $70 \mathrm{~mm}$ distance between the upward and downward shanks. The BHs are different since they are filled with various grouting materials such as sand of different coarseness, and finished with a bentonite layer at the top in order to avoid intrusion of pollutants into the BH, as described in Ground-Med (2011).

Regarding the ground thermal properties, some laboratory analyses were performed on soil samples. Those analyses provided values of $1.43 \mathrm{~W} / \mathrm{mK}$ for the thermal conductivity and $2250 \mathrm{~kJ} / \mathrm{m}^{3} \mathrm{~K}$ for the 
volumetric heat capacity. A high uncertainty (around 20\%) was found in the estimation of the ground thermal conductivity, confirmed with a thermal response test, which resulted in $1.6 \mathrm{~W} / \mathrm{mK}$, (Montagud et al., 2011). The undisturbed ground temperature and the $\mathrm{BH}$ resistance at the facility are around $18.5^{\circ} \mathrm{C}$ and $0.118 \mathrm{mK} / \mathrm{W}$, (Urchueguía, 2006). The reliability of the latter two values is uncertain.

Temperatures, mass flow rates and power consumption were measured during operation of the system. For the temperature, four-wire Pt100 resistance thermometers with inaccuracy $\pm 0.1^{\circ} \mathrm{C}$ are used. The mass flow meters are Danfoss Coriolis meters, model MASS 6000 with signal converter Compact IP 67 and inaccuracy $< \pm 0.1 \%$.Two temperature sensors are placed at the inlet and outlet of the heat pump. Additionally, there are sensors placed at the inlet and outlet of each $\mathrm{BH}$, and seven sensors are measuring ground temperatures at different depths in three of the six BHs. Data obtained from this sensor network are registered every minute and collected by a data acquisition unit Agilent HP34970A with plug-in modules HP34901A. The temperature of the fluid is obtained from the average of the inlet and outlet temperature of all BHEs and corrected by a factor that accounts for the switching on/off time of the heat pump. The temperatures are registered every minute and grouped in daily values for the measurements presented in this paper.

In order to analyze the collected data and calculate all the parameters used for the characterization of the system's performance, a tool was developed using Excel macros. The heat rate injected or extracted from the ground can be calculated from the inlet and outlet water temperatures, the mass flow rate, and the circulating fluid properties. The thermal loads at the installation are provided every minute and these are averaged in order to obtain hourly loads. For the purpose of this work, the hourly loads will be grouped in daily load steps, obtained as the average of the hourly loads for each day.

\subsection{The thermal process at the UPV Demo site}

The numerical model at the UPV Demo site is built according to the geometrical characteristics of the BH field, as listed in Table 4. The thermal properties of the ground are defined considering the values of lab measurements and their uncertainty. Thus, in the present study, the thermal conductivity is defined as $1.6 \mathrm{~W} / \mathrm{mK}$, the volumetric heat capacity is $2250 \mathrm{~kJ} / \mathrm{m}^{3} \mathrm{~K}$, and the $\mathrm{BH}$ thermal resistance is 0.118 $(\mathrm{mK} / \mathrm{W})$ as described in Section 5.1.

Table 4 Geometrical Aspect Ratios- UPV Demo site

\begin{tabular}{cl}
\hline Geometrical Aspect Ratio & Value \\
\hline$D / H$ & 0.074 \\
$r_{b} / H$ & 0.0016 \\
$B / H$ & 0.063 \\
\hline
\end{tabular}

The thermal response of the ground is studied by considering two different cases, defined as "Constant q" and "Uniform T". The difference between these is basically how the BC is set at the BH wall. The "Constant q" model assumes a constant heat flux along the BH length and the model defined as "Uniform T" takes into account that the BHs are thermally connected imposing a uniform temperature along the $\mathrm{BH}$ length, and the total heat flow to the BH field is held constant.

Regarding the methodology to study the case of constant heat flux as $\mathrm{BC}$ at the $\mathrm{BH}$ wall, it is defined by applying a heat flux $\mathrm{BC}$ at the wall of each BH, as described in (Monzó et al. 2013a). The uniform temperature $\mathrm{BC}$ has been applied as described in Section 3.2 in this paper.

Both "Constant q" and "Uniform T" models are first validated against reference g-functions. The reference solutions are the same as used in Section 4: "FLS-Constant q", "FLS-Uniform T" and "FDMUniform T". Figure 10 shows the g-functions generated with our numerical model, named as "FEM- 
Constant q" and "FEM- Uniform T" in accordance with the BC set at the BH wall, including the aforementioned reference g-functions.

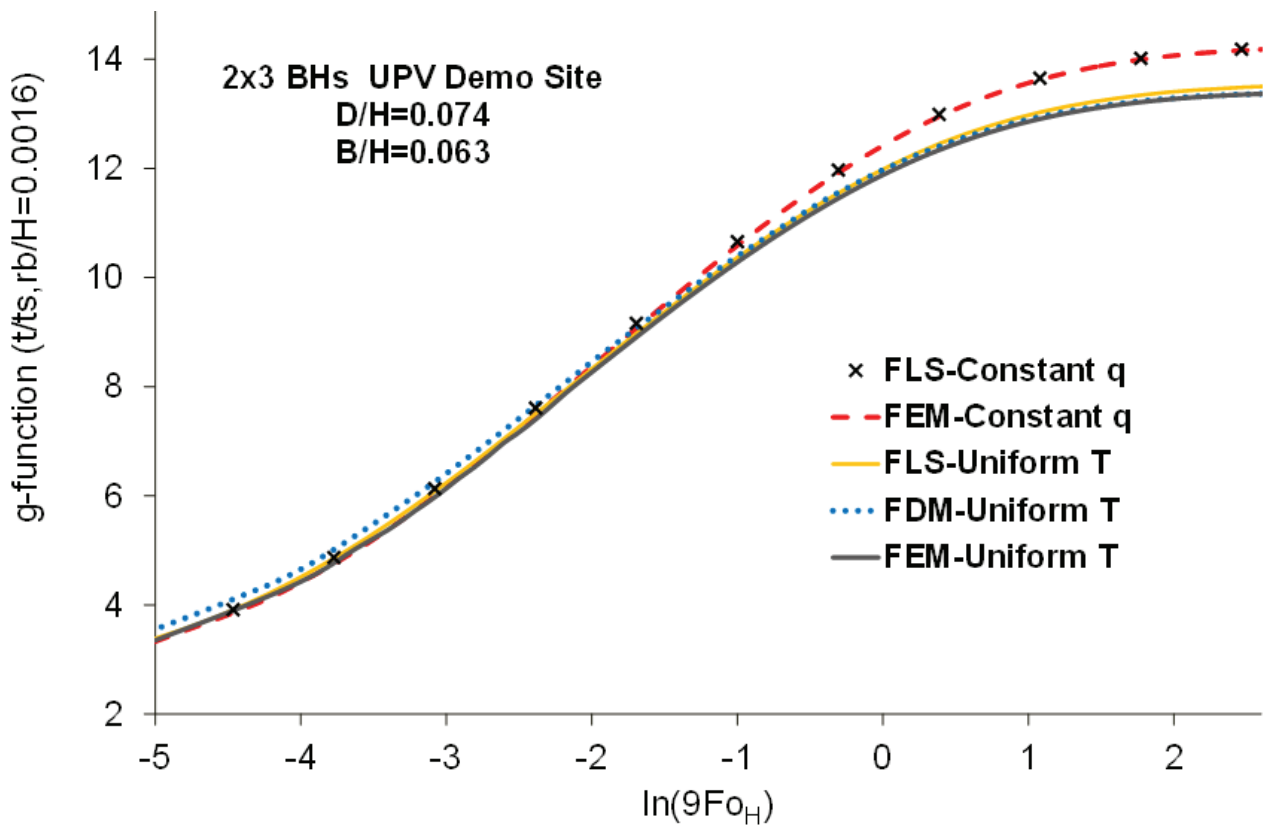

Figure 10 : Validation of the numerical model at UPV Demo site: g-functions generation and comparison with its reference solutions.

The g-function obtained from "FEM- Constant q" agrees, as expected, with the "FLS-Constant q" generated g-function. It can also be observed that all g-functions except the "FDM-Uniform T" present a similar short term behavior. From times equivalent to $\ln \left(9 \mathrm{Fo}_{\mathrm{H}}\right) \approx-2$, i.e. about 550 days for the UPV Demo site characteristics, the implications of using the different BCs are clearly observed in the resulting gfunctions. In the beginning, the g-function from the "FDM-Uniform T" presents higher values than those generated from both "FEM-Constant q" and "FEM-Uniform T" models. The differences between "FDMUniform T" and "FEM-Uniform T" are about 0.2 units. Translating these into temperature and time dimensions for a case of $20 \mathrm{~W} / \mathrm{m}$ constant injection, the temperature differences between "FDM-Uniform $\mathrm{T}$ " and "FEM-Uniform $\mathrm{T}$ " is $0.39 \mathrm{~K}$, after about 45 days $\left(\ln \left(9 \mathrm{FO}_{\mathrm{H}}\right)=-4.5\right)$. These deviations are partly related to an approximation, probably an interpolation (Yang et al., 2010; Malayappan and Spitler, 2013), that the software carries out on the g-functions contained in the library in order to obtain an approximate solution for the specific geometrical characteristics of the UPV Demo site. The differences are also partly attributed to the new parameters accounted for the g-function generation, especially the ratio $\mathrm{D} / \mathrm{H}$. The gfunction obtained from the "FEM-Uniform T" model increases $1.59 \%$ when the ratio D changes from 3.5 to 4.5 meters and $\mathrm{H}$ is $47.5 \mathrm{~m}$, when the $\ln \left(9 \mathrm{Fo}_{\mathrm{H}}\right)$ is equal to 2 . This also shows that the influence of $\mathrm{D}$ over $\mathrm{H}$ becomes more significant when the active borehole length decreases in comparison with the deviation presented in the g-function of $2 \times 3$ BHs configuration presented in Section 4.2. This influence was also shown in (Cimmino et al., 2013).The differences are also partly attributed to some adjustable features on the mesh in our numerical model.

In the asymptotic part of the curves (after about $\ln \left(9 \mathrm{Fo}_{\mathrm{H}}\right)>2$, that is around 82 years for the UPV Demo site characteristics), the g-function "FEM-Uniform T" presents very similar values to "FDMUniform T", whereas the g-function from the "FEM-Constant q" model overestimates it.

In comparison with "FLS-Uniform T" the "FEM-Uniform T" comes out in slightly lower values. The deviations starts around $\ln \left(9 \mathrm{Fo}_{\mathrm{H}}\right)=-4$ and are about $0.9 \%$ in the remaining time period.

Once the models are validated, the daily fluid temperatures are simulated for the input daily loads measured at UPV. In both models, "Constant q" and "Uniform T", the input data corresponds to measured 
daily loads during 6 years, estimated as described above. The initial condition is set to the undisturbed temperature of the ground. For the simulations, it has been defined as the annual average ambient temperature in Valencia, which is about $19.5^{\circ} \mathrm{C}$, somewhat higher than presented in Urchueguía (2006). A Thermal Response Test (TRT) result would likely be the optimum input, since it gives a more accurate value of the temperature in the ground in situ. Moreover, a temperature $\mathrm{BC}$ equal to $19.5^{\circ} \mathrm{C}$ is set at the outer boundary of the BH field domain. The geothermal heat flux is not taken into account in this case.

The computational mesh is identical to the one described in the numerical model section in "Constant q" and "Uniform T" models. Radial elements are applied in the surrounding of the BHs. The smallest elements are chosen in the proximity of the BHs, their size increasing with the distance from the BHs. Tetrahedral elements are chosen in the surrounding domain located under the BH field, which is about 200x249 $\mathrm{m}$ in the horizontal plane with $200 \mathrm{~m}$ depth and in the inactive upper part (having thickness D according to Table 4) at the top of the BH field. Concerning the solver, its features are specified according to the input data. Special treatment has been taken with regards to the time stepping of the solver calculations. Since the input comprises heating and cooling loads, the duration of the loads becomes a restriction for the maximum time stepping of the calculation. By computing the solution, the $\mathrm{BH}$ temperatures are generated for the two different models considered in this paper.

Finally, the temperature of the fluid is calculated by assuming the average $\mathrm{BH}$ thermal resistance presented in the previous section. The fluid temperatures resulting from both simulations, "Constant q" and "Uniform T" models, are compared with the temperatures measured at the UPV Demo site as well as with a calculation based on the commercial software EED, labeled as Commercial Software. To calculate the monthly fluid temperatures in the commercial software, average monthly loads are repeatedly applied during 6 years.

\subsection{Simulation of Daily Temperatures}

Although the simulation was carried out for 6 years of operation, only the results of the sixth year are presented in this paper. Figure 11 shows the comparison of the simulated and measured fluid temperatures. The measured values are labeled as "Measured Daily Temp.". The values obtained from the simulation are referred to as "Simulated Daily Temp.-Constant q" and "Simulated Daily Temp.-Uniform $\mathrm{T}$ " for the "Constant q" and "Uniform $\mathrm{T}$ " models, respectively. The monthly temperature values calculated with the aforementioned commercial software are labeled as "Simulated Monthly Temp.Commercial Software". The daily average heat flow is also presented as a bar graph in Figure 11.

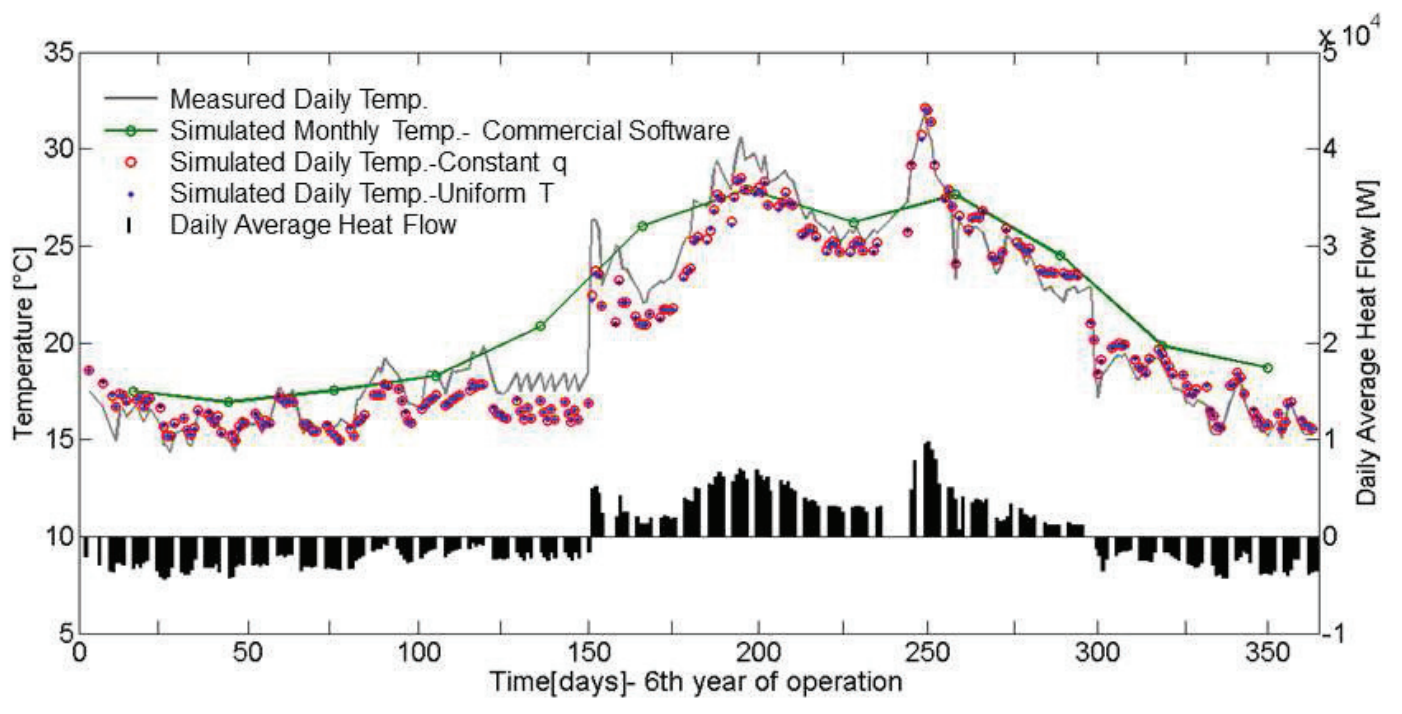


Figure 11 : Daily average heat flow and fluid temperatures. Measured and simulated values during the 6th year of operation.

As observed in Figure 11, the differences between the simulated fluid temperatures in "Constant q" and "Uniform T" models are virtually insignificant. However, the differences should be more pronounced in imbalanced compact BH fields and after longer time, in which the "FEM- Uniform T" model, which accounts for the thermal interactions between the BHs, may give a better approximation to the reality, as shown in (Malayappan and Spitler, 2013) and (Monzó et al., 2013b).. In comparison with the measured values, there is better agreement in heating (heat drawn from the ground) than during the cooling period in both cases. With regards to the simulated monthly values, the commercial software gives a simplified description of the operation during most of the period and also seems to overestimate the temperatures to some extent, which is perhaps related to the fact that the average monthly loads over the whole simulation period have been repeated.

The average temperature difference between the measured and simulated values is about $0.5 \mathrm{~K}$, varying within a range of -1 and $2 \mathrm{~K}$. There are also a few points that fall way outside the trend lines and they are attributed to errors in the measurements. The differences become greater in cooling periods as shown in Figure 11. This result indicates that the simulation is not fully representative for the actual prescribed input properties at the site. A change in the undisturbed ground temperature or the $\mathrm{BH}$ resistance, as well as the inclusion of the geothermal heat flux would shift the simulated temperatures. The simulated results could likely be improved by adjusting some input parameters.

\section{CONCLUSIONS}

A novel numerical model to simulate the thermal response of borehole fields is described in this paper. This model pays special attention to the boundary condition $(\mathrm{BC})$ at the borehole $(\mathrm{BH})$ wall and its realization in time and space. A hypothetical Highly Conductive Material (HCM) inserted into the $\mathrm{BH}$ is used to simulate a uniform temperature condition at the BH wall. The HCM is extended up to a bar thermally connecting all the BHs within the BH field, representing the hydraulic connection within single BHs connected in parallel. The flexibility of this model allows the study of the thermal response of any $\mathrm{BH}$ field configuration imposing a variety of temperature conditions at the $\mathrm{BH}$ wall with a simple change of the thermal conductivity of the HCM. Although this model presently has a limited practical application, it can be used as a research tool to either generate g-functions for specific situations or to improve accuracy or handle situations that other methods cannot handle. Examples are spatial variations in the ground thermal properties and irregular $\mathrm{BH}$ fields.

In order to illustrate the application of this approach, g-functions are first generated for a single $\mathrm{BH}$ and for a $2 \times 3$ BHs configuration. The results are compared with the solution from SBM (uniform temperature) and FLS approaches. In general, the results show a good agreement with the "SBM" and a recent version of the FLS solution, producing slightly lower g-values than these. The deviation becomes somewhat higher in the asymptotic part of the g-function. When comparing with SBM in a single BH configuration, the deviation in g-values varies from 0.04 to 0.07 units for short and long times, respectively. In the case of a $2 \times 3 \mathrm{BHs}$ configuration, the deviation is 0.16 and 0.30 units in the beginning and the asymptotic part of the curve, respectively. Temperature and linear heat flux profiles along the depth at different time instants confirm the existence of the uniform temperature $\mathrm{BC}$ at the wall of all BHs, showing details about the three dimensional heat transfer process in the $\mathrm{BH}$ field. Future work will be devoted to address the inaccuracies of the method regarding the mesh features.

The $2 \times 3$ model is also used to generate daily fluid temperatures for real daily loads over 6 years of operation and compared with measured data. The model is built according to the geometrical characteristics and ground thermal properties of a $2 \times 3 \mathrm{BH}$ field located at the UPV. Two different BCs at the $\mathrm{BH}$ wall are tested separately, constant heat flux and uniform temperature. The simulated daily values obtained from both BCs approaches show a good agreement with the measured temperatures. Due to the 
fact that the system is almost fully thermally balanced over the year, i.e. the yearly cooling and heating loads are nearly the same, the differences between the "Constant q" and "Uniform T" approaches become insignificant. However, the "Uniform T" model may present better agreement than "Constant q" model for imbalanced compact BH fields and after longer time. Future work will be also devoted to assess the differences in the $\mathrm{BC}$ at $\mathrm{BH}$ wall for imbalanced compact $\mathrm{BH}$ fields. The best agreement with the measured data is found during the heating periods, where the differences are within a $0.5 \mathrm{~K}$ range all the time. The authors will also dedicate extra work to have a better prediction of the fluid temperature by improving the model itself, as mentioned above, and adjusting the input parameters. Moreover, the authors will address the uncertainties due to the repetition of the monthly loads year-to-year by superimposing the monthly variable loads to the g-function generated from the "FEM-Uniform T"

\section{ACKNOWLEDGMENTS}

The Seventh Research Framework Programme "Advanced ground source heat pump systems for heating and cooling in Mediterranean climates" Ground-Med TREN/FP7EN/218895 is acknowledged for partly supporting this project. The Swedish Research Council FORMAS and EFFSYS+ are also acknowledged for financing this research.

\section{REFERENCES}

Andersson, O. and Bjelm, L. 2013. Geothermal energy use, country update for Sweden, Proceeding of European Geothermal Congress 2013, EGC2013-CUR30, Pisa, Italy, June.

Claesson, J. and Javed, S. 2011. An analytical method to calculate borehole fluid temperatures for timescales from minutes to decades. ASHRAE Transactions, vol. 117(2), pp.279-288.

Cimmino, M., Bernier, M., and Adams, F. 2013. A contribution towards the determination of g-functions using the finite line source. Applied Thermal Engineering 51: 101-412

Cimmino, M. and Bernier, M. 2013. Preprocessor for the generation of g-functions used in the simulation of geothermal systems. Proceedings of BS2013: $13^{\text {th }}$ Conference of international Building Performance Simulation Association, Chambéry, France, August.

Cimmino, M. and Bernier, M. 2014. A semi-analytical method to generate g-functions for geothermal bore fields. International Journal of Heat and Mass Transfer 70: 641-650

Corberán, J.M., Finn, D.P., Montagud, C., Murphy, F.T., and Edwards, K.C. 2011. A quasi-steady state mathematical model of an integrated ground source heat pump for building space control. Energy and Buildings 43: 82-92.

Cullin, J., Montagud, C., Ruiz-Calvo, F. and Spitler, J.D. 2013. Experimental validation of ground heat exchanger design methodologies using real monitored data. ASHRAE Transactions, vol. 120(2):357369

Cullin, J. 2014. Advancements in the simulations of ground source heat pump systems. $\mathrm{PhD}$ Thesis. School of Mechanical and Aerospace Engineering, Oklahoma State University.

Cullin, J., Spitler, J.D, Montagud, C., Ruiz-Calvo, F., Rees, S., Naicker, S., Konečný, P. and Southard, L. 2015. Validation of vertical ground heat exchanger design methodologies. Science and Technology for the Built Environment. 21(2). In press.

Eskilson, P. 1986. Superposition Borehole Model. Manual for Computer Code, Dept. of Mathematical Physics, Lund Institute of Technology, Lund, Sweden.

Eskilson, P. 1987. Thermal analyses of heat extraction Boreholes. PhD thesis. Dept. of Mathematical Physics, Lund Institute of Technology, Lund, Sweden.

Fossa, M. 2011a. A fast method for evaluating the performance of complex arrangements of borehole heat exchangers. HVAC\&R 17:6 948-958.

Fossa, M. 2011b. The temperature penalty approach to the design of borehole heat exchangers for heat pump Applications. Energy and Buildings 43: 1473-1479. 
Ground-Med 2011 D.6.5: University Polytechnic of Valencia demo system in Spain. Work Package 6: Integrated ground source heat pump demonstration systems. Seventh FP No TREN/FP7EN/218895. http://www.groundmed.eu/uploads/media/Deliverable_6.5_UPV_demo_system_01.pdf 24/04/2012.

Hellström, G. and Sanner, B. 1994. Earth Energy Designer: software for dimensioning of deep boreholes for heat extraction. Proceedings of Calorstock- $6^{\text {th }}$ International Conference on Thermal Energy Storage, Espoo/Helsinki, Finland

Lamarche, L. and Beauchamp, B. 2007. A new contribution to the finite line-source model for geothermal boreholes. Energy and Buildings 39: 188-198.

Lazzarotto, A. 2014. A network-based methodology for the simulation of borehole heat storage systems. Renewable Energy 62: 265-275.

Malayappan, V. and Spitler, J.D. 2013 Limitations of using heat flux assumptions in sizing vertical borehole heat exchanger fields. Proceeding of Clima 2013. June 16-19. Prague.

Marshall, C.L. and Spitler, J.D. 1994. GLHEPRO -The professional ground loop heat exchanger design software, Users guide, School of Mechanical and Aerospace Engineering, Oklahoma State University, Stillwater, Oklahoma.

Montagud, C., Corberán, J.M., Montero, Á. and Urchueguía, J.F. 2011. Analysis of the energy performance of a ground source heat pump system after five years of operation. Energy and Buildings 43: 3618-3626.

Monzó, P., Acuña, J., Fossa, M. and Palm, B. 2013a. Numerical generation of the temperature response factors for a borehole heat exchanger field. European Geothermal Congress, ISBN 978-2-8052-02261, EGC2013-SG118, Pisa, Italy.

Monzó, P., Acuña, J., Mogensen P. and Palm, B. 2013b. A study of the thermal response of a borehole field in winter and summer. Proceedings of 5th International Conference on Applied Energy, ICAE2013-524, Pretoria, South Africa.

Urchueguía, J. 2006. Curso de doctorado sobre "Técnicas Energéticas Avanzadas". Universitat Politècnica de València.

Yang, H., Cui, P. and Fang, Z. 2010. Vertical-borehole ground-coupled heat pumps: A review of models and systems. Applied Energy 87: 16-27.

Zeng, H., Diao, N. and Fang, Z. 2002. A finite line-source model for boreholes in geothermal heat exchangers. Heat Transfer - Asian Research 31: 558-567. 